\title{
Race modifies the relationship between cognition and Alzheimer's disease cerebrospinal fluid biomarkers
}

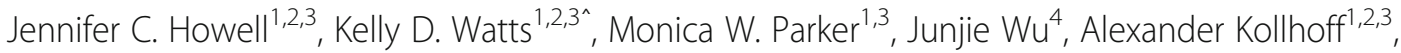

Thomas S. Wingo ${ }^{1,2,3}$, Cornelya D. Dorbin ${ }^{1,3}$, Deqiang Qiu ${ }^{3,4}$ and William T. Hu $u^{1,2,3^{*}}$

\begin{abstract}
Background: African Americans have been reported to have a higher prevalence of Alzheimer's disease (AD) than Caucasians, but etiology-specific AD biomarkers have not been systematically analyzed in older African Americans. Coexisting cerebrovascular disease may also contribute to this increased prevalence. We hypothesized that cerebrospinal fluid (CSF) biomarkers of amyloid, neurodegeneration, and endothelial dysfunction would differ between older African Americans and Caucasians with normal cognition and cognitive impairment associated with AD.

Methods: We prospectively recruited 135 older Americans to undergo detailed clinical, neuropsychological, genetic, magnetic resonance imaging (MRI), and CSF analysis from 2013 to 2015 at Emory University (Atlanta, GA, USA). We compared levels of CSF markers for $\beta$-amyloid (Aß42, A 440$)$, total and phosphorylated tau (t-tau and $p$-tau 181 , respectively), endothelial dysfunction (soluble vascular cell adhesion molecule 1, soluble intercellular adhesion molecule 1), a-synuclein, and neurodegeneration (neurofilament light chain [NfL]), as well as MRI markers, for hippocampal atrophy and cerebrovascular disease (white matter hyperintensity [WMH] volume).
\end{abstract}

Results: Sixty-five older African Americans (average age, 69.1 years) and 70 older Caucasians (average age, 70.8 years) were included. After adjusting for demographic variables, AD risk alleles, and cognitive function, older African Americans had lower CSF levels of p-tau ${ }_{181}$ (difference of $7.4 \mathrm{pg} / \mathrm{ml} ; 95 \% \mathrm{Cl}, 3.7-11.2 \mathrm{pg} / \mathrm{ml} ; p<0.001$ ), t-tau (difference of $23.6 \mathrm{pg} / \mathrm{ml} ; 95 \% \mathrm{Cl}, 9.5-37.7 ; p=0.001$ ), and $\mathrm{A} \beta 40$ (difference of $1.35 \mathrm{ng} / \mathrm{ml} ; 95 \% \mathrm{Cl}, 0.29-2.42 \mathrm{ng} / \mathrm{ml} ; p=0.013$ ) despite similar levels of A 342 , NfL, WMH volume, and hippocampal volume. Cognitively impaired African Americans also had lower CSF t-tau/Aß42 (difference of 0.255 per 1-SD change in composite cognition; 95\% Cl, 0.100-0.409; $p=0$. 001 ) and $p$-tau ${ }_{181} / A \beta 42$ (difference of 0.076 per 1-SD change in composite cognition; $95 \% \mathrm{Cl}, 0.031-0.122 ; p=0.001$ ). These could not be explained by measured biomarkers of non-AD processes, but African Americans may be more susceptible than Caucasians to the cognitive effects of WMH.

Conclusions: Despite comparable levels of CSF A 42 and A $42 / A \beta 40$, cognitive impairment in African Americans is associated with smaller changes in CSF tau markers but greater impact from similar WMH burden than Caucasians. Race-associated differences in CSF tau markers and ratios may lead to underdiagnosis of AD in African Americans.

Trial registration: ClinicalTrials.gov, NCT02089555. Retrospectively registered on 14 March 2014.

Keywords: African American, Amyloid, Dementia, Endothelial dysfunction, Mild cognitive impairment, Tau

\footnotetext{
* Correspondence: wthu@emory.edu

Deceased

'Department of Neurology, Emory University School of Medicine, 615

Michael Street, 505F, Atlanta, GA 30322, USA

${ }^{2}$ Center for Neurodegenerative Diseases, Emory University School of

Medicine, Atlanta, GA, USA

Full list of author information is available at the end of the article
} 


\section{Background}

Multiple studies have shown quantitative and qualitative differences in Alzheimer's disease (AD) between African Americans and Caucasians. At the population level, the prevalence of $\mathrm{AD}$ dementia is nearly doubled in African Americans compared with Caucasians [1, 2]; African Americans are more likely than Caucasians to have multiple members with dementia within the same family [3]; and genome-wide association studies have identified genetic variants uniquely associated with $\mathrm{AD}$ in African Americans (e.g., $A B C A 7$ [4-6], COBL [7]). Cognitively, African Americans with $\mathrm{AD}$ are more likely than Caucasians to have nonamnestic profiles [8] and slower decline [9]. At autopsy, African Americans with AD also have greater ischemic and Lewy body copathology and perhaps less transactive response DNA-binding protein $43 \mathrm{kDa}$ (TDP-43) pathology $[10,11]$. Together, these findings point to a possible AD endophenotype in African Americans, although few epidemiologic or genome-wide association studies have addressed the accuracy of clinical AD diagnosis. This is especially relevant if a greater proportion of African Americans than Caucasians with vascular dementia are misdiagnosed with $\mathrm{AD}$, which can potentially be corrected through antemortem biomarkers associated with amyloid, tau, and vascular pathology. However, African American recruitment into modern AD biomarker studies such as the Alzheimer's Disease Neuroimaging Initiative (ADNI) has traditionally been limited [12], and there are few longitudinal clinicopathologic studies involving African Americans that have provided autopsy-based information.

We hypothesized that etiologic biomarkers for AD such as cerebrospinal fluid (CSF) protein profiles can improve the antemortem characterization of $\mathrm{AD}$ and non-AD pathology in older African Americans [13, 14]. Because of interindividual heterogeneity in cognition, genetic risks, and comorbidities, enrolling African Americans at their population prevalence $(\sim 13 \%)$ is likely insufficiently powered to detect race-associated differences in most biomarker studies. Therefore, we prospectively recruited similar numbers of older African Americans and Caucasians with normal cognition, mild cognitive impairment (MCI), and AD dementia to undergo detailed clinical, neuropsychological, magnetic resonance imaging (MRI), genetic, and CSF analysis [15]. We characterized the cohort's CSF amyloid ( $\beta$-amyloid $1-40$ and $1-42$ [A $\beta 40$ and $A \beta 42$, respectively]), tau (total tau and tau phosphorylated at threonine 181 [t-tau and $\mathrm{p}$-tau $\mathrm{tan}_{181}$, respectively]), neurodegenerative (neurofilament light chain [NfL]), and candidate endothelial markers (soluble vascular cell adhesion molecule 1 [sVCAM-1]; soluble intercellular cell adhesion molecule 1 [sICAM-1]) $[16,17]$ to test their associations with cognitive impairment within each race.

\section{Methods}

Ethics, consent, and permission

This study was approved by the Emory Institutional Review Board. Informed consent was obtained from all subjects or their authorized representatives.

\section{Subjects}

We enrolled 65 older African Americans and 70 older Caucasians into a cross-sectional study at Emory University from 1 July 2013 to 30 June 2015. Based on our preliminary data, this sample size was sufficient to detect a difference in CSF t-tau levels. Recruitment for this study was previously described [15]. Briefly, potential participants were identified through the Emory Alzheimer's Disease Research Center, the Emory Cognitive Neurology Clinic, or community forums on aging and memory, and individuals were contacted if there was a subjective report of normal cognition or a diagnosis of MCI or AD dementia. Study protocols, including CSF and MRI analysis, were discussed with potential participants, and those diagnosed with non-AD dementia (e.g., vascular, dementia with Lewy bodies, frontotemporal dementia or primary progressive aphasia, normal pressure hydrocephalus) were excluded from the study. Subjects with MCI with features suggestive of non-AD pathology (e.g., rapid eye movement sleep behavior disorder) were also excluded. A total of 288 subjects were contacted, and 135 (47\%) consented to participate. The study completion rate was $98 \%$ for MRI (133 of 135) and 93\% for lumbar puncture (126 of 135). Subjects who did not undergo lumbar puncture were similar to those who did with regard to age, sex, race, education, and apolipoprotein $\mathrm{E}(A P O E)$ and $A B C A 7$ genotypes. All biochemical and MRI analyses were performed by experienced operators blinded to the subject's race and cognitive functioning.

\section{Clinical and neuropsychological characterization}

Each participant underwent a detailed interview so that we could obtain demographic information, including selfreported race (Caucasians of Hispanic or Latino ethnicity were not included in this study), vascular risk factors (coronary artery disease, congestive heart failure, atrial fibrillation, hypertension, hyperlipidemia, diabetes, suspected transient ischemic attack), other medical comorbidities (e.g., cancer), and medications (e.g., use of angiotensinconverting enzyme inhibitors or angiotensin II receptor blockers). Each subject also underwent a detailed neurologic examination and neuropsychological analysis for assessment of function in cognitive domains. These included (1) memory (Consortium to Establish A Registry for Alzheimer's Disease word list delayed recall, Brief Visual Memory Test-Revised [BVMT-R] delayed recall), (2) executive function (Trail Making Test B, reverse digit span [RD], Symbol Digit Substitution Test, and letter-guided fluency), (3) language (Boston Naming Test [60 items], category fluency), 
and (4) visuospatial function (Judgment of Line Orientation [JOLO], Rey-Osterrieth complex figure test). With the exception of BVMT-R, JOLO, and RD, subtest Z-scores were calculated according to published normative data, adjusting for age, sex, education, and race $[18,19]$. Domain-specific Z-scores were calculated by averaging subtest Z-scores, and $Z$-scores for the four domains were averaged to generate composite cognitive Z-scores. This approach was used in previous longitudinal multiracial studies, including ADNI, and it allows for domain-specific and global assessment of cognition [20-23]. Each participant was then assigned a diagnosis according to consensus criteria (Table 1), including those for normal cognition, $\mathrm{MCI}[24,25]$, and $\mathrm{AD}$ dementia (global Clinical Dementia Rating 1 or 2) [26, 27].
Linear regression analysis among subjects with normal cognition showed that BVMT-R scores were not affected by race $(p=0.207)$. African American subjects' performance on JOLO was significantly better than published norms [28], so a regression analysis was used to develop Z-scores for African Americans. This showed that JOLO scores correlated with sex, race, and education $(4.05 \times \operatorname{sex}[1=$ male; $0=$ female $]+0.60 \times$ years of education $+1.94 \times$ race $[1=$ Caucasian; $0=$ African American]). RD scores were also influenced by race $(1.297 \times$ race $[1=$ Caucasian; $0=$ African American]). Mean and SD values among subjects with normal cognition were then calculated after adjusting for these variables to obtain Z-scores. Cognitively impaired subjects suspected of having a non-AD dementia (vascular, Lewy

Table 1 Baseline characteristics of African American and Caucasian research participants

\begin{tabular}{|c|c|c|c|c|c|c|c|c|}
\hline & \multicolumn{2}{|l|}{ Overall } & \multicolumn{2}{|l|}{ Normal cognition } & \multicolumn{2}{|l|}{$\mathrm{MCl}$} & \multicolumn{2}{|l|}{$A D$} \\
\hline & $\begin{array}{l}\text { African American } \\
(n=65)\end{array}$ & $\begin{array}{l}\text { Caucasian } \\
(n=70)\end{array}$ & $\begin{array}{l}\text { African American } \\
(n=27)\end{array}$ & $\begin{array}{l}\text { Caucasian } \\
(n=29)\end{array}$ & $\begin{array}{l}\text { African American } \\
(n=27)\end{array}$ & $\begin{array}{l}\text { Caucasian } \\
(n=25)\end{array}$ & $\begin{array}{l}\text { African American } \\
(n=11)\end{array}$ & $\begin{array}{l}\text { Caucasian } \\
(n=16)\end{array}$ \\
\hline Age, years (SD) & $69.1(7.4)$ & $70.8(7.7)$ & $67.5(6.2)$ & $71.4(8.1)$ & $67.7(7.6)$ & $71.5(5.8)$ & $71.4(9.1)$ & $68.5(9.4)$ \\
\hline Male sex, n (\%) & 29 (45\%) & $29(41 \%)$ & 10 (37\%) & $12(41 \%)$ & 15 (56\%) & $10(40 \%)$ & $4(36 \%)$ & 7 (44\%) \\
\hline $\begin{array}{l}\text { Education, years } \\
\text { (SD) }\end{array}$ & $16.1(2.8)$ & $16.4(2.7)$ & $15.8(2.7)$ & $17.0(2.6)$ & $16.3(2.9)$ & $17.1(2.6)$ & $16.7(3.4)$ & $14.2(1.9)$ \\
\hline MMSE (SD) & $26.1(4.0)$ & $26.7(3.6)$ & $28.0(1.8)$ & $28.9(0.8)$ & $26.6(2.4)$ & $27.6(1.7)$ & $20.5(5.9)$ & $21.4(3.4)$ \\
\hline Hypertension & $47(72 \%)^{a}$ & $32(46 \%)$ & $17(63 \%)$ & $13(45 \%)$ & $21(78 \%)$ & $14(56 \%)$ & 9 (82\%) & 5 (31\%) \\
\hline Diabetes & $22(34 \%)^{a}$ & $4(6 \%)$ & $9(33 \%)$ & $2(7 \%)$ & $8(30 \%)$ & 0 & $5(45 \%)$ & $2(12 \%)$ \\
\hline $\begin{array}{l}\text { Average number } \\
\text { of vascular risk } \\
\text { factors (SD) }\end{array}$ & $1.9(1.2)^{\mathrm{a}}$ & $1.3(1.1)$ & $1.5(1.1)$ & $1.3(1.3)$ & $2.2(1.3)$ & $1.4(0.9)$ & $2.2(1.2)$ & $1.0(1.0)$ \\
\hline $\begin{array}{l}\text { Having at least } \\
\text { one } A P O E \text { \&4 } \\
\text { allele, } n(\%)\end{array}$ & $31 / 60(52 \%)$ & $35 / 70(50 \%)$ & $7 / 25$ (29\%) & $11(38 \%)$ & $15 / 26(58 \%)$ & $13(52 \%)$ & 9/10 (90\%) & $11(69 \%)$ \\
\hline $\begin{array}{l}\text { Having } A B C A 7 \\
\text { risk allele, } n(\%)\end{array}$ & $28 / 60(47 \%)^{a}$ & 16/70 (23\%) & $12 / 25(48 \%)$ & $8(27 \%)$ & $13 / 25(52 \%)$ & $6(24 \%)$ & $3 / 10(30 \%)$ & $2(12 \%)$ \\
\hline $\begin{array}{l}\text { Completed LP, } \\
n(\%)\end{array}$ & 58 (89\%) & 68 (97\%) & $23(85 \%)$ & $28(97 \%)$ & 25 (93\%) & 25 (100\%) & 10 (91\%) & 15 (94\%) \\
\hline \multicolumn{9}{|l|}{ CSF } \\
\hline $\begin{array}{l}\mathrm{A} \beta 42, \mathrm{pg} / \mathrm{ml} \\
\text { (SD) }\end{array}$ & $212.3(118)$ & $207.2(148)$ & $273(110)$ & $250(131)$ & $178(110)$ & $226(172)$ & $158(105)$ & $96(61)$ \\
\hline $\begin{array}{l}\mathrm{A} \beta 40, \mathrm{ng} / \mathrm{ml} \\
\text { (SD) }\end{array}$ & $7.89(2.92)$ & $9.29(3.32)$ & $7.21(1.96)^{a}$ & $10.03(3.65)$ & $7.83(3.21)$ & $9.38(3.18)$ & $9.49(3.61)$ & $7.91(2.54)$ \\
\hline $\begin{array}{l}\text { t-Tau, } \mathrm{pg} / \mathrm{ml} \\
\text { (SD) }\end{array}$ & $47.0(31.1)^{\mathrm{a}}$ & $71.5(47.8)$ & $36.3(12.0)^{a}$ & $58.6(29.0)$ & $46.4(38.6)$ & $66.6(38.4)$ & $72.8(27.5)$ & $103.8(72.9)$ \\
\hline $\begin{array}{l}\mathrm{p}-\mathrm{Tau}_{181}, \mathrm{pg} / \\
\mathrm{ml}(\mathrm{SD})\end{array}$ & $17.9(9.3)^{a}$ & $25.6(12.6)$ & $13.8(5.1)^{\mathrm{a}}$ & $22.5(9.8)$ & $18.8(10.2)$ & $24.0(12.0)$ & $25.3(10.3)$ & $34.2(14.9)$ \\
\hline $\begin{array}{l}\text { CSF t-Tau/Aß42> } \\
0.39\end{array}$ & 17/58 (29\%) & $31 / 68(46 \%)$ & $2 / 23(9 \%)$ & $7 / 28(25 \%)$ & 9/25 (36\%) & $13 / 25(52 \%)$ & $6 / 10(60 \%)$ & $11 / 15(73 \%)$ \\
\hline $\begin{array}{l}\log _{10}(\mathrm{NfL}), \mathrm{ng} / \mathrm{ml} \\
(\mathrm{SD})\end{array}$ & $2.87(0.27)$ & $2.97(0.19)$ & $2.76(0.23)$ & $2.96(0.22)$ & $2.87(0.26)$ & $2.96(0.15)$ & $3.14(0.23)$ & $3.02(0.22)$ \\
\hline $\begin{array}{l}\log _{10}(\mathrm{WMH}) \\
\mathrm{cm}^{3}(\mathrm{SD})\end{array}$ & $0.367(0.453)$ & $0.437(0.364)$ & $0.238(0.337)$ & $0.355(0.365)$ & $0.401(0.515)$ & $0.483(0.308)$ & $0.607(0.466)$ & $0.514(0.431)$ \\
\hline
\end{tabular}

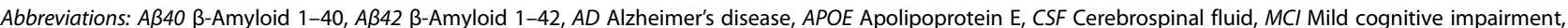
MMSE Mini Mental State Examination, NfL Neurofilament light chain, $p$-Tau ${ }_{181}$ Tau phosphorylated at threonine 181, $t$-Tau Total tau, WMH White matter hyperintensity

Values shown are unadjusted

a Values or proportions differed between the two races $(p<0.005)$ 
body, and frontotemporal dementia) were excluded. Because we aimed to examine the relationship between race and cognitive function and the sensitivity of CSF t-tau/ A $342>0.39$ for AD derived from a previous autopsy study [29] is not $100 \%$, we did not exclude $\mathrm{MCI}$ and $\mathrm{AD}$ subjects whose CSF t-tau/A $\beta 42$ ratio was $<0.39$.

\section{MRI acquisition and analysis}

MRI data were acquired using a MAGNETOM Trio, A Tim System 3.0-T scanner (Siemens Healthcare, Erlangen, Germany), including 3D T1-weighted magnetizationprepared rapid acquisition with gradient echo anatomical imaging (repetition time/inversion time/echo time, 2300/ 900/3.0 milliseconds; flip angle, 9 degrees; voxel size, $1 \times$ $1 \times 1.2 \mathrm{~mm}^{3} ; 176$ slices). Cortical and subcortical volumes were extracted by employing a user-independent parcellation process in FreeSurfer (version 5.03; Massachusetts General Hospital/Harvard University, Boston, MA, USA) running on the CentOS 6.6 operating system [30]. Subcortical white matter and deep gray matter structures are parcellated by combining data from voxel intensity, probabilistic atlas locations, and local relationships between anatomical structures. Total white matter hyperintensity (WMH) volume was derived from T2-weighted fluidattenuated inversion recovery sequences as previously described [31]. WMH volume was not normally distributed and was therefore log-transformed for statistical analysis.

\section{CSF collection and analysis}

CSF samples were collected between 8:00 a.m. and 2:00 p.m. without requiring fasting. This window was chosen because CSF A $\beta 42$ levels during these times represent approximately $95-105 \%$ of average A 342 over time [32]. CSF was immediately aliquotted after collection and before freezing, and otherwise we used the ADNI biofluid protocols, including the use of 24-gauge Sprotte needles, aspiration syringes, and transfer into 15-ml polypropylene tubes. To avoid measurement bias related to well positions, sample positions were randomized for each biomarker assay.

CSF biomarkers were measured following the manufacturers' protocols: $\mathrm{A} \beta 42$, $\mathrm{t}$-tau, and $\mathrm{p}$-tau ${ }_{18}$ levels were measured using the INNO-BIA AlzBio3 immunoassay (Fujirebio, Malvern, PA, USA) in a Luminex 200 platform (Luminex, Austin, TX, USA) [33] (average interplate coefficients of variation of $13 \%$ for $A \beta 42,10 \%$ for $t$-tau, and $11 \%$ for $\mathrm{p}$-tau $\left.{ }_{181}\right), \mathrm{A} \beta 40$ levels using the INNOTEST ${ }^{\oplus}$ enzymelinked immunosorbent assay (ELISA) (Fujirebio), $\alpha$ synuclein levels using the Novex ${ }^{\circledR}$ ELISA (Thermo Fisher Scientific, Waltham, MA, USA), NfL levels using the NFlight ${ }^{\ominus}$ ELISA (Uman Diagnostics, Umeå, Sweden), and sICAM-1 and sVCAM-1 levels using a commercial multiplex kit (MILLIPLEX ${ }^{\ominus}$ MAP Human Neurodegenerative
Magnetic Bead Panel 3 [HNDG3MAG-36 K]; EMD Millipore, Billerica, MA, USA).

\section{DNA extraction and analysis}

DNA was extracted from buffy coat as previously described [34]. Single-nucleotide polymorphism (SNP) genotyping for $A P O E$ and $A B C A 7$ was performed using TaqMan $^{\curvearrowleft}$ assays (Thermo Fisher Scientific). ABCA7 SNP rs3764650 (catalogue number 4351379; Thermo Fisher Scientific) was analyzed because of its association with AD [6] and because it is in linkage disequilibrium with SNP rs115550680 implicated in AD in African Americans [4]. Statistical analysis was restricted to those with successful genotyping ( $n=124$ cases; all had CSF, 123 had MRI).

\section{Statistical analysis}

Statistical analysis was performed using IBM SPSS version 22.0 software (IBM, Armonk, NY, USA). Baseline demographic variables and $\mathrm{AD}$ risk alleles were compared between African Americans and Caucasians within each diagnostic category (normal cognition, $\mathrm{MCI}$, and $\mathrm{AD}$ ) using the chi-square test or Fisher's exact test for categorical variables and Student's $t$ test for continuous variables, with $p<$ 0.005 to adjust for multiple comparisons. Among subjects with normal cognition, all biomarkers were normally distributed except for NfL and WMH, which were then $\log _{10^{-}}$ transformed. Analysis of covariance (ANCOVA) was used to determine race-associated differences in CSF AD biomarkers (t-tau, p-tau ${ }_{181}, A \beta 40, t-t a u / A \beta 42, p-$ tau $_{181} / A \beta 42$, $\mathrm{A} \beta 42 / \mathrm{A} \beta 40), \alpha$-synuclein, and $\mathrm{p}$-tau $\mathrm{ta1}_{181}$-to-t-tau ratio $(\mathrm{p} / \mathrm{t}$ tau), adjusting first for cognitive functioning (diagnostic category, Mini Mental State Examination [MMSE], or cognitive Z-score). Race and the interaction between race and cognitive function were both included in these models. When appropriate, age, sex, APOE, ABCA7 risk allele status, and CSF A 342 levels were further included in the ANCOVA. F-statistic and $p$ values for main effects were reported, along with coefficient estimate and 95\% CI after stepwise removal of factors and covariates with $p>0.10$. To account for coexisting cerebrovascular risks, hypertension, diabetes, and WMH volume were then added. Furthermore, sVCAM-1 was analyzed first through Pearson's correlational analysis and then in its own ANCOVA to determine its suitability as a CSF endothelial marker. Finally, because African Americans had lower CSF tau markers than Caucasians but similar WMH volumes, linear regression analysis was used to model potential interactions between race and tau and between race and WMH volume to determine if cognition was more affected in African Americans than in Caucasians on the basis of the same unit of change in CSF tau markers or WMH volume. In this model, composite cognitive Z-score was the dependent variable, with race, sex, having the APOE $\varepsilon 4$ allele, having an ABCA7 risk allele, hypertension, and diabetes as 
categorical variables and age, education, $A \beta 42$, $t$-tau, $\log _{10}$ (WMH), and education as continuous variables. Independent variables were entered in a stepwise fashion to achieve a model consisting of only main effects. Race and education were not significant factors in this model, because the cognitive Z-scores had been adjusted for these two factors, but sex and age remained significant to account for variance from the sex- and age-adjusted cognitive Z-scores. To test the hypothesis that race modified the relationship between cognition and $\mathrm{t}$-tau or $\mathrm{WMH}$, an interaction term of $\mathrm{t}$ tau $\times$ race or $\log (\mathrm{WMH}) \times$ race was added to the model.

\section{Results}

\section{Baseline and genetic characteristics}

Among the 135 participants, African Americans were more likely than Caucasians to have the $A B C A 7$ risk allele $(46.7 \%$ vs. $22.9 \%, p=0.005)$ and the ICAM1 Lys56Met polymorphism (33.3\% vs. $5.7 \%, p<0.001)$ (Table 1$)$. African Americans were also more likely to have hypertension ( $72 \%$ vs. $46 \%, p=0.003)$ and diabetes $(34 \%$ vs. $6 \%, p<0.001$ ) (Table 1). The two groups were otherwise similar in age, sex, education, proportion with the APOE $\varepsilon 4$ allele, and other vascular risk factors (Table 1 ).

\section{Relationship between race and CSF biomarkers for amyloid and tau}

As a group (regardless of diagnosis), African Americans had lower CSF levels of $\mathrm{p}$-tau 181 (17.9 vs. $25.6 \mathrm{pg} / \mathrm{ml}, p<$ 0.001 ) (Fig. 1a), t-tau (47.0 vs. $71.5 \mathrm{pg} / \mathrm{ml}, p=0.001$ ) (Fig. 1b), and A $\beta 40$ levels (7.88 vs. $9.29 \mathrm{ng} / \mathrm{ml}, p=0.017$ ) (Fig. 1d) than Caucasians, but they had similar A $\beta 42$ levels (Fig. 1c). Univariate analysis of these biomarkers revealed the greatest differences in subjects with normal cognition (Table 1), but the relationship between biomarker levels and demographic variables necessitated the use of ANCOVA to determine whether race influenced CSF biomarker levels in a cognition-dependent or cognitionindependent fashion. We found that, independent of cognitive functioning (data for cognitive Z-scores presented; diagnostic category and MMSE produced similar results), age, sex, APOE $\varepsilon 4$ and ABCA7 risk alleles, and A 342 levels, African Americans had lower levels of t-tau [difference of $23.6 \mathrm{pg} / \mathrm{ml}$; 95\% CI, 9.5-37.7; $F(2,122)=$ $10.99 ; p=0.001$ ], p-tau 181 levels [difference of $7.4 \mathrm{pg} / \mathrm{ml}$; 95\% CI, 3.7-11.2 pg/ml; $F(2,122)=15.79 ; p<0.001]$, and A 340 [difference of $1.355 \mathrm{ng} / \mathrm{ml} ; 95 \% \mathrm{CI}, 0.293-2.417 \mathrm{ng} /$ $\mathrm{ml} F(2,122)=6.385 ; p=0.013]$. Race also affected the ratio biomarker of t-tau/A $\beta 42$ (Fig. 1e) and p-tau ${ }_{181} / A \beta 42$ (not shown) according to cognitive functioning, but it had minimal effect on $A \beta 42 / A \beta 40$ (Fig. 1f). For both tau markers, cognitively impaired African Americans had lower CSF ttau/A 342 [difference of 0.255 per 1 -SD change in composite cognition; 95\% CI, 0.100-0.409; $F(2,122)=10.67 ; p=$ 0.001 ] and $p$-tau ${ }_{181} / \mathrm{A} \beta 42$ [difference of 0.076 per 1 -SD change in composite cognition; 95\% CI, 0.031-0.122; $F(2,122)=10.94 ; p=0.001]$ ratios than cognitively impaired Caucasians.

\section{CSF neurodegenerative and endothelial markers do not account for race-associated AD biomarker differences}

Lower t-tau and p-tau ${ }_{181}$ levels in African Americans may be explained by misdiagnosis, less neurodegeneration, or greater contribution from non-AD copathology toward cognitive impairment. Because CSF A $\beta 42$ levels were indistinguishable between African Americans and Caucasians, cognitively impaired subjects in the two racial groups were equally likely to have $\beta$-amyloidopathy, which makes misdiagnosis less probable. To determine if there was a difference in neurodegeneration between the two races, we examined the relationship between race, CSF NfL levels, and hippocampal atrophy because the latter two are influenced by $\mathrm{AD}$ as well as non-AD disorders. ANCOVA showed that cognitively normal African Americans had lower $\log (\mathrm{NfL})$ than cognitively normal Caucasians $[F(2,122)=3.525 ; p=0.017]$, but $\log (\mathrm{NfL})$ did not differ between the two races for those with cognitive impairment. Race also had no effect on hippocampal volumes $[F(2,122)=1.78 ; p=0.185]$ to suggest less neurodegeneration in cognitively impaired African Americans. Similarly, among those with CSF ttau/A 342 not consistent with $\mathrm{AD}$, race had no effect on CSF $\alpha$-synuclein levels [implicated in Lewy body disease; $F(2,75)=1.078 ; p=0.303$ ] or $\mathrm{p} / \mathrm{t}$-tau ratio [implicated in frontotemporal lobar degeneration with TDP-43 immunoreactive inclusions; $F(2,75)=0.775 ; p=0.382$ ]

Because African Americans had a higher prevalence of hypertension and diabetes than Caucasians in our cohort (Table 1), cerebrovascular disease could account for cognitive impairment in the setting of lower CSF tau levels. Including hypertension, diabetes, and total WMH volume in the ANCOVA did not change the effect of race on p-tau ${ }_{181}$ $[F(2,121)=18.13 ; p<0.001)$, t-tau $[F(2,121)=6.790 ; p=$ $0.010]$, or $\mathrm{A} \beta 40$ levels $[F(2,121)=5.084 ; p=0.008]$. Therefore, vascular risk factors and total WMH volume do not sufficiently account for the CSF AD biomarker differences between the two races. At the same time, peripheral vascular risk factors may not adequately reflect cerebrovascular disease burden. We thus tested whether CSF levels of two candidate endothelial markers (sVCAM-1 and sICAM-1) could be used as surrogate markers of endothelial dysfunction. As a proof of principle, we first analyzed the relationship between these two markers and race, peripheral vascular risks, and WMH among cognitively normal subjects enriched for those without AD pathology (CSF A $\beta 42$, $>192 \mathrm{ng} / \mathrm{ml} ; n=34)$. At the univariate level, CSF sVCAM-1 levels, but not sICAM-1 levels, correlated positively with older age $(R=0.514 ; p<0.001)$, Caucasian race $(R=0.35 ; p$ $=0.026$ ), higher WMH volume (log-transformed; $R=0.433$; 

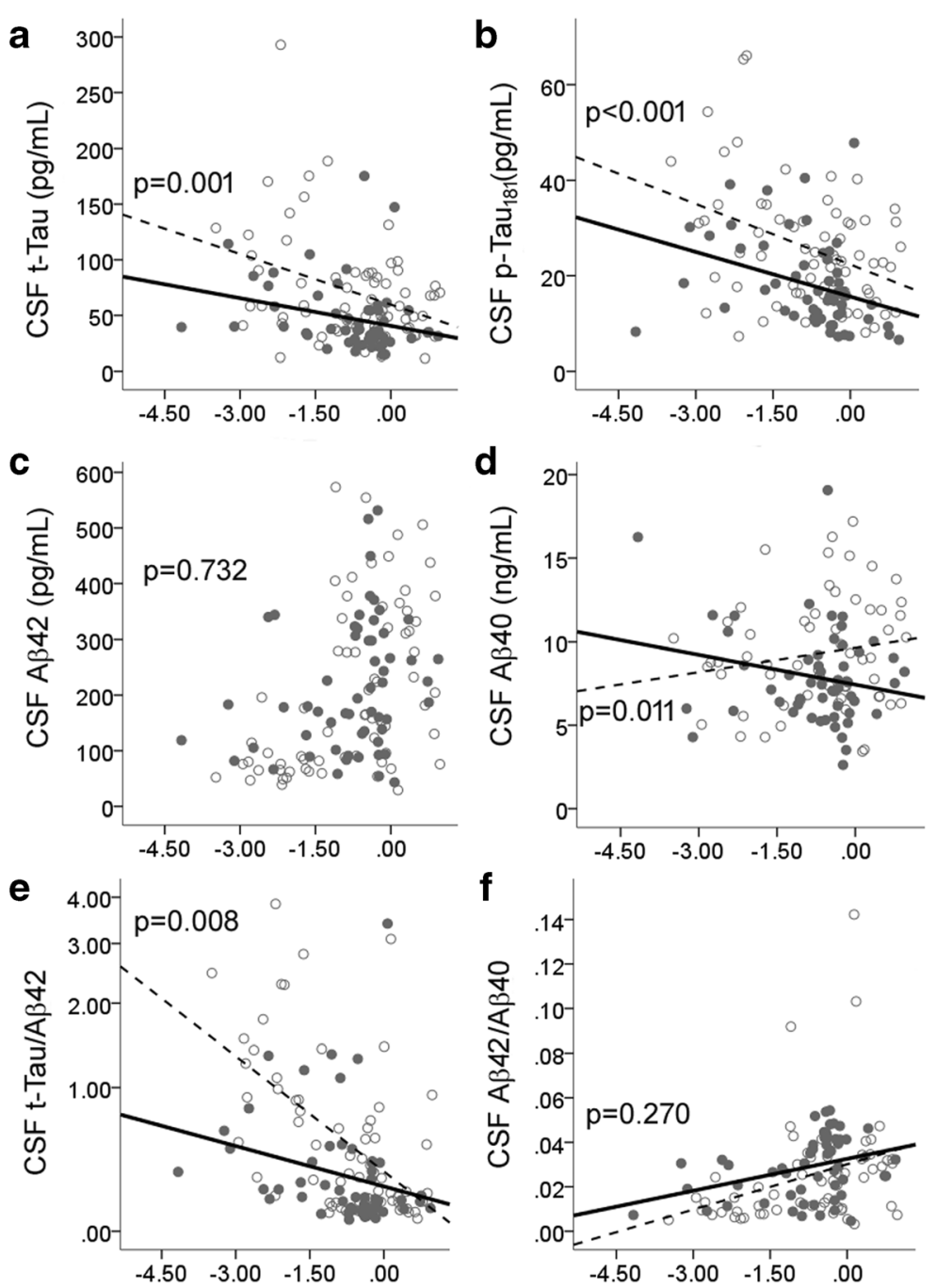

f

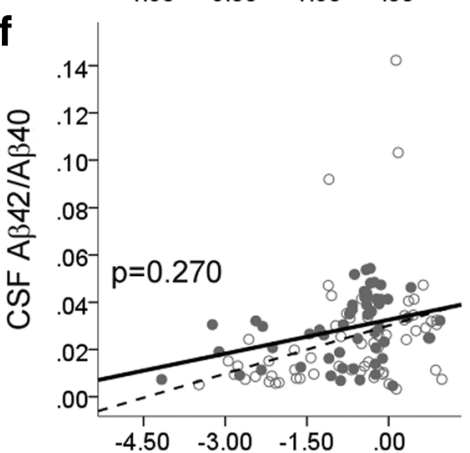

Composite Cognitive Z-score

Composite Cognitive Z-score

Caucasian

- African American

Fig. 1 Cerebrospinal fluid (CSF) levels of tau and amyloid markers in older African Americans and Caucasians according to cognitive function. Composite cognitive $Z$-scores are shown on the $x$-axis (lower score corresponds to worse cognitive function). African Americans (closed circles) had lower CSF levels of total tau (t-tau) (a), tau phosphorylated at threonine 181 (p-tau $\left.{ }_{181}\right)(\mathbf{b})$, and $\beta$-amyloid 1-40 (A 340$)$ (d) than Caucasians (open circles). Raw values are shown, with dashed lines representing trends among Caucasians and solid lines representing trends among African Americans. The differences persisted after adjusting for age, sex, apolipoprotein E (APOE) and ABCA7 genotypes, and $\beta$-amyloid 1-42 (Aß42) levels (c), which did not differ between the two groups. CSF biomarker t-tau/Aß42 ratio was lower in African Americans than in Caucasians when there was cognitive impairment (e), but race did not have a significant effect on CSF biomarker A $342 / A \beta 40$ (f)

$p=0.005)$, greater number of vascular risk factors $(R=$ $0.357 ; p=0.020)$, history of congestive heart failure $(R=$ $0.444 ; p=0.003)$, and history of atrial fibrillation $(R=0.399$; $p=0.009)$. ANCOVA showed that race modified the relationship between sVCAM-1 levels and WMH volume (Fig. 2a) and between sVCAM-1 levels and vascular risk (Fig. 2b). Similar results were obtained when all subjects with CSF $\mathrm{t}$-tau/Ab42 $<0.39$ were included (Fig. 2c, d). In sum, whereas sVCAM-1 correlated with known vascular disease markers in Caucasians, it is a poor measure of endothelial dysfunction in African Americans.

\section{Race modifies the impact of WMH on cognition}

To reconcile our finding that, after controlling for raceadjusted cognitive performance, African Americans had lower CSF tau marker levels than Caucasians despite similar levels of WMH, NfL, and hippocampal volumes, we tested if race modified the relationship between cognition and the two intermediate etiologic biomarkers (tau, WMH). To test this hypothesis, we determined, using multivariate linear regression analysis, whether there was an interaction between race and each of these markers of $\mathrm{AD}\left(\mathrm{t}\right.$-tau, $\left.\mathrm{p}-\mathrm{tau}_{181}\right)$ or vascular (WMH) 


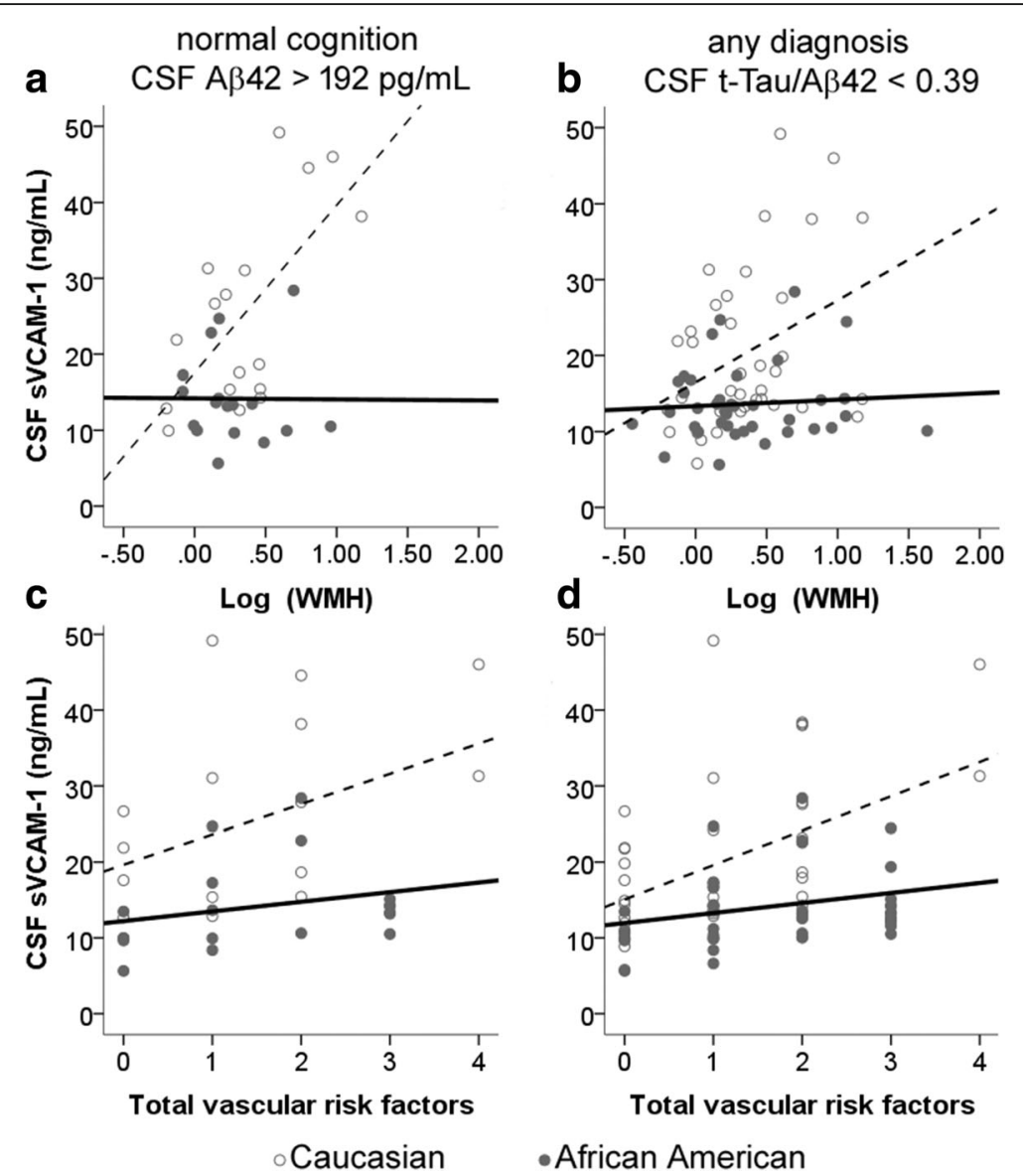

Fig. 2 Relationship between cerebrospinal fluid (CSF) soluble vascular cell adhesion molecule 1 (sVCAM-1) levels and other vascular markers according to race. In Caucasians (open circles), CSF sVCAM-1 levels strongly correlated with log-transformed white matter hyperintensity (WMH) volumes derived by magnetic resonance imaging $(\mathbf{a}, \mathbf{b})$ and the total number of peripheral vascular risk factors $(\mathbf{c}, \mathbf{d})$ whether a more $(\mathbf{a}, \mathbf{c})$ or less stringent $(\mathbf{b}$, $\mathbf{d})$ threshold was applied to identify subjects with no Alzheimer's disease pathology. However, there was no such correlation in African Americans (closed circles). Aß42 B-Amyloid 1-42, t-Tau Total tau

pathology to influence cognition (see the Methods section above). At the univariate level, cognition was influenced by $A P O E$ and $A B C A 7$ genotypes as well as by $\log _{10}(\mathrm{WMH})$ and CSF levels of $\mathrm{A} \beta 42$ and $\mathrm{t}$-tau. Introducing the interaction term of race $\times \log _{10}(\mathrm{WMH})$ showed that the interaction term had a stronger effect on cognitive Z-scores than $\log _{10}(\mathrm{WMH})$ alone (Table 2), and that African American race was associated with greater cognitive impairment than Caucasian race for every unit of change in $\log _{10}(\mathrm{WMH})$. This was not the case for tau or $\mathrm{p}-\mathrm{tau}_{181}$.

\section{Discussion}

Although multicenter efforts such as ADNI have advanced the characterization of $\mathrm{AD}$ in terms of cognitive decline, fluid and imaging biomarkers, and genetic variants, whether findings from these homogeneous cohorts can be applied to a more diverse population remains unknown. African Americans represent the largest racial minority group in the United States, and clinicopathologic studies linking their phenotypes to underlying pathology on autopsy or pathology-associated biomarkers are rare. To the best of our knowledge, this is the first study to directly identify race to modify CSF AD biomarker levels and the relationships between WMH and cognition in a small but well-characterized cohort of older African Americans and Caucasians. Whereas race did not affect the primary CSF marker of amyloid deposition (Aß42 levels), race was associated with lower CSF $\mathrm{p}-\mathrm{tau}_{181}$ and t-tau levels in African Americans, independent of cognition, and it affected the AD biomarkers $\mathrm{t}$-tau/A $\beta 42$ and $\mathrm{p}$-tau $\mathrm{ta1}_{181} / \mathrm{A} \beta 42$ according to cognitive impairment. Race also affected $A \beta 40$ levels, but it did not influence $\mathrm{AD}$ biomarker $\mathrm{A} \beta 42 / \mathrm{A} \beta 40$. We propose that these differences did not result from less neurodegeneration or greater endothelial dysfunction in African Americans. Instead, our model suggests that the same degree of WMH had a greater impact on cognition in 
Table 2 Demographic and biomarker variables that influence cognitive Z-scores

\begin{tabular}{lll}
\hline Variables & $\mathrm{B}(95 \% \mathrm{Cl})$ & $p$ Value \\
\hline Intercept & $-3.08(-4.70,-1.45)$ & $<0.001$ \\
Log(WMH) & $-0.35(-0.89,0.19)$ & 0.206 \\
Male sex & $0.54(0.21,0.87)$ & 0.002 \\
Having at least one APOE \&4 allele & $-0.36(-0.71,-0.01)$ & 0.042 \\
Having ABCA7 risk allele & $0.38(0.04,0.72)$ & 0.03 \\
A 442 & $0.003(0.001,0.004)$ & $<0.001$ \\
t-Tau & $-0.006(-0.010,-0.002)$ & 0.003 \\
Age & $0.039(0.015,0.063)$ & 0.002 \\
African American race $\times \log (\mathrm{WMH})$ & $-0.496(-1.074,0.081)$ & 0.091 \\
\hline
\end{tabular}

Abbreviations: A $A 42 \beta$-Amyloid 1-42, APOE Apolipoprotein E, $t$-Tau Total tau, $W M H$ White matter hyperintensity

A stepwise regression model was used to determine factors most strongly associated with cognitive function as reflected by cognitive Z-scores. African American race had a trend of worsening cognitive functioning for the same degree of change in WMH. If the log(WMH) term was removed from this model, African American race was associated with lower cognitive Z-score (by $0.496 ; p=0.006$ ) per unit change of $\log (\mathrm{WMH})$. Race and sex were not significant factors in this model. Similar results were obtained when $\mathrm{p}$-tau ${ }_{181}$ instead of $\mathrm{t}$-tau was entered into the model

African Americans than in Caucasians through an as yet unknown mechanism.

Assessing cognitive decline in minority populations has been challenging because of discrepancies between various normative datasets $[28,35,36]$. In keeping with others' experiences, established normative data underestimated the cognitive functioning of older African Americans in our cohort, possibly owing to their generally high level of education. Objective etiologic biomarkers have the potential of identifying $\mathrm{AD}$ and other neurodegenerative diseases early in the disease course, independent of language, cultural, and socioeconomic confounds. Our observation of a race effect on three common AD biomarkers- $t$-tau, $\mathrm{p}$ $\mathrm{tau}_{181}$, and $\mathrm{A} \beta 40$ - underscores the biological and clinical importance of biomarker studies in minority populations. We believe that cognitive impairment in African Americans was indeed associated with $\beta$-amyloidopathy, because CSF A $\beta 42$ levels in subjects with $\mathrm{MCI}$ or dementia did not differ between the two races. These findings imply that using existing cutoff values for CSF biomarker levels or ratios involving tau derived from largely Caucasian populations may lack sensitivity in identifying $\mathrm{AD}$ in minority populations, and it is not known if cerebral tau imaging can overcome this. We extended these observations by exploring multiple CSF and MRI markers involved in neurodegeneration, and we found race-independent (e.g., NfL) as well as race-dependent (sVCAM-1) markers. However, a key observation was the absence of a difference in CSF $\mathrm{A} \beta 42$ levels and WMH volumes between the two races. According to the model of sequential biomarker changes in $\mathrm{AD}$ [37], more modest increases in t-tau or p-tau ${ }_{181}$ levels in cognitively impaired African Americans might result in lower CSF NfL levels and greater hippocampal volumes than similarly impaired Caucasians. This was not the case, which led us to search for an alternative explanation for the relatively greater neurodegenerative changes (measured by NfL and hippocampal volumes with less specificity to AD) in African Americans associated with more modest tau changes. Exactly how WMH may result in greater neurodegeneration and cognitive deficits in African Americans is unknown. WMH is thought to represent terminal organ damage from chronic ischemia, and it is possible that African Americans have a greater degree of subclinical endothelial dysfunction than Caucasians. It is also possible that differences in the brain structural (as measured by white matter tract integrity) and functional (as measured by resting state connectivity) networks predispose African Americans to the effects of ischemia. Finally, a synergistic effect between WMH and brain amyloid burden has been reported for cognition [38], and brain amyloid deposition in African Americans may preferentially enhance WMH-associated neurotoxicity over tau-associated neurodegeneration. Future studies should prospectively test each of these hypotheses to identify potentially modifiable factors in an effort to reduce the raceassociated increase in AD prevalence [39].

Our model was suggestive of a process involving WMH to account for lower tau marker levels in African Americans, but other explanations need to be considered. There still exists the possibility of greater non-AD pathology in African Americans, because CSF $\alpha$-synuclein and $p / t$-tau are imperfect markers of Lewy body and TDP-43 pathology [40]. Although amyloid and tau PET imaging may provide additional information, more detailed neuropathologic characterization may be necessary to definitively determine the $\mathrm{AD}$ pathology in African Americans with clinical AD dementia. Variants in the MAPT or other genes (besides $A P O E$ or $A B C A 7$ ) may also influence $\mathrm{t}$-tau levels or the relative levels of different tau isoforms. The $\mathrm{H} 2$ haploptype of MAPT is rare among African, Asian, and Native American populations [41], but a previous study failed to identify any $M A P T$ variant to associate with CSF t-tau or p-tau ${ }_{181}$ levels among those without evidence of brain amyloid deposition [42]. Other limitations of the study include the lack of an independent validation cohort, not using genetic markers to ascertain African ancestry [43] or to account for additional race-related AD risks [7], limited information on environmental exposure, and inclusion of only the most commonly studied $\mathrm{AD}$ and endothelial markers. It is also worth emphasizing that genomic African ancestry is inadequate to biologically or sociologically characterize aging and disease in African Americans, as pointed out by many of our participants and colleagues, and this study's findings may not translate to other African American cohorts of different genetic and socioeconomic backgrounds. 


\section{Conclusions}

In sum, we report that CSF tau biomarker levels are strongly influenced by race in a cohort of older African Americans and Caucasians in Atlanta. This has important implications for the use of diagnostic biomarkers derived from CSF and potentially other modalities (e.g., positron emission tomography) in unique populations underrepresented in existing large biomarker studies. Our data also raise the possibility that African American race may modify the relationship between an MRI marker of cerebrovascular disease and cognition, likely through an APOE-independent mechanism. There is an urgent need to test the generalizability of these findings in more cohorts of African Americans.

\section{Abbreviations}

AD: Alzheimer's disease; ADNI: Alzheimer's Disease Neuroimaging Initiative; ANCOVA: Analysis of covariance; APOE: Apolipoprotein E; A 340 : $\beta$-Amyloid 1-40; Aß42: $\beta$-Amyloid 1-42; BVMT-R: Brief Visual Memory Test-Revised; CSF: Cerebrospinal fluid; ELISA: Enzyme-linked immunosorbent assay; JOLO: Judgment of Line Orientation; MCl: Mild cognitive impairment; MMSE: Mini Mental State Examination; MRI: Magnetic resonance imaging; NfL: Neurofilament light chain; $\mathrm{p}-\mathrm{Tau}_{181}$ : Tau phosphorylated at threonine 181; RD: Reverse digit span; sICAM-1: Soluble intercellular adhesion molecule 1; SNP: Single-nucleotide polymorphism; sVCAM-1: Soluble vascular cell adhesion molecule 1; TDP-43: Transactive response DNA-binding protein 43 kDa; t-Tau: Total tau; WMH: White matter hyperintensity

\section{Acknowledgements}

The authors acknowledge the following persons for assistance with enrollment: James Lah, MD, PhD; Allan Levey, MD, PhD; Chadwick Hales, MD, PhD; Angela Ashley, MD; Felicia Goldstein, PhD; and Andrea Kippels, CNP.

\section{Funding}

This work was supported by National Institutes of Health grants AG43885, AG42856, and AG25688.

\section{Availability of data and materials}

The datasets used and/or analyzed during the present study are available from the corresponding author on reasonable request.

\section{Authors' contributions}

WTH, MWP, and DQ were responsible for the conception and design of the study. WTH, JCH, KDW, MWP, JW, AK, TSW, CDD, and DQ were responsible for acquisition, analysis, and interpretation of data. WTH, JCH, KDW, MWP, and DQ were responsible for drafting the manuscript and revising it critically for important intellectual content. All authors read and approved the final manuscript.

\section{Ethics approval and consent to participate}

This study was approved by the Emory Institutional Review Board (number 66145). Informed consent was obtained from all subjects or their authorized representatives.

\section{Consent for publication}

Not applicable.

\section{Competing interests}

WTH has a patent (assignee, Emory University) on the use of CSF p/t-tau ratio in the evaluation of frontotemporal lobar degeneration, has received research support from Avid Pharmaceuticals, and has received travel support from Eli Lilly and Hoffman-La Roche. DQ has received research support from Medtronic and Siemens Healthcare.

\section{Publisher's Note}

Springer Nature remains neutral with regard to jurisdictional claims in published maps and institutional affiliations.

\section{Author details}

${ }^{1}$ Department of Neurology, Emory University School of Medicine, 615 Michael Street, 505F, Atlanta, GA 30322, USA. ${ }^{2}$ Center for Neurodegenerative Diseases, Emory University School of Medicine, Atlanta, GA, USA. ${ }^{3}$ Alzheimer's Disease Research Center, Emory University School of Medicine, Atlanta, GA, USA. ${ }^{4}$ Department of Radiology, Emory University School of Medicine, Atlanta, GA, USA.

Received: 28 July 2017 Accepted: 16 October 2017

Published online: 02 November 2017

\section{References}

1. Association A's. 2010 Alzheimer's disease facts and figures. Alzheimers Dement. 2010;6:158-94.

2. Manly JJ, Tang MX, Schupf N, Stern Y, Vonsattel JP, Mayeux R. Frequency and course of mild cognitive impairment in a multiethnic community. Ann Neurol. 2008;63:494-506

3. Green RC, Cupples LA, Go R, Benke KS, Edeki T, Griffith PA, Williams M, Hipps Y, Graff-Radford N, Bachman D, Farrer LA. Risk of dementia among white and African American relatives of patients with Alzheimer disease. JAMA. 2002;287:329-36.

4. Reitz C, Jun G, Naj A, Rajbhandary R, Vardarajan BN, Wang LS, Valladares O, Lin CF, Larson EB, Graff-Radford NR, et al. Variants in the ATP-binding cassette transporter (ABCA7), apolipoprotein $\mathrm{E} \varepsilon 4$, and the risk of late-onset Alzheimer disease in African Americans. JAMA. 2013;309:1483-92.

5. Logue MW, Schu M, Vardarajan BN, Buros J, Green RC, Go RC, Griffith P, Obisesan TO, Shatz R, Borenstein A, et al. A comprehensive genetic association study of Alzheimer disease in African Americans. Arch Neurol. 2011:68:1569-79.

6. Hollingworth P, Harold D, Sims R, Gerrish A, Lambert JC, Carrasquillo MM, Abraham R, Hamshere ML, Pahwa JS, Moskvina V, et al. Common variants at ABCA7, MS4A6A/MS4A4E, EPHA1, CD33 and CD2AP are associated with Alzheimer's disease. Nat Genet. 2011:43:429-35.

7. Mez J, Chung J, Jun G, Kriegel J, Bourlas AP, Sherva R, Logue MW, Barnes LL, Bennett DA, Buxbaum JD, et al. Two novel loci, COBL and SLC10A2, for Alzheimer's disease in African Americans. Alzheimers Dement. 2017;13:119-29.

8. Livney MG, Clark CM, Karlawish JH, Cartmell S, Negron M, Nunez J, Xie SX, Entenza-Cabrera F, Vega IE, Arnold SE. Ethnoracial differences in the clinical characteristics of Alzheimer's disease at initial presentation at an urban Alzheimer's disease center. Am J Geriatr Psychiatry. 2011:19:430-9.

9. Barnes LL, Wilson RS, Li Y, Aggarwal NT, Gilley DW, McCann JJ, Evans DA. Racial differences in the progression of cognitive decline in Alzheimer disease. Am J Geriatr Psychiatry. 2005;13:959-67.

10. Graff-Radford NR, Besser LM, Crook JE, Kukull WA, Dickson DW. Neuropathologic differences by race from the National Alzheimer's Coordinating Center. Alzheimers Dement. 2016;12:669-77.

11. Barnes LL, Leurgans S, Aggarwal NT, Shah RC, Arvanitakis Z, James BD, Buchman AS, Bennett DA, Schneider JA. Mixed pathology is more likely in black than white decedents with Alzheimer dementia. Neurology. 2015;85:528-34.

12. Petersen RC, Aisen PS, Beckett LA, Donohue MC, Gamst AC, Harvey DJ, Jack Jr $C R$, Jagust WJ, Shaw LM, Toga AW, et al. Alzheimer's Disease Neuroimaging Initiative (ADNI): clinical characterization. Neurology. 2010;74:201-9.

13. Hu WT, Chen-Plotkin A, Arnold SE, Grossman M, Clark CM, Shaw LM, Pickering E, Kuhn M, Chen Y, McCluskey L, et al. Novel CSF biomarkers for Alzheimer's disease and mild cognitive impairment. Acta Neuropathol. 2010;119:669-78.

14. Clark CM, Schneider JA, Bedell BJ, Beach TG, Bilker WB, Mintun MA, Pontecorvo MJ, Hefti F, Carpenter AP, Flitter ML, et al. Use of florbetapir-PET for imaging beta-amyloid pathology. JAMA. 2011;305:275-83.

15. Howell JC, Parker MW, Watts KD, Kollhoff A, Tsvetkova DZ, Hu WT. Research lumbar punctures among African Americans and Caucasians: perception predicts experience. Front Aging Neurosci. 2016;8:296.

16. Hall JR, Wiechmann AR, Johnson LA, Edwards M, Barber RC, Winter AS, Singh M, O'Bryant SE. Biomarkers of vascular risk, systemic inflammation, and microvascular pathology and neuropsychiatric symptoms in Alzheimer's disease. J Alzheimers Dis. 2013;35:363-71.

17. Ewers M, Mielke MM, Hampel H. Blood-based biomarkers of microvascular pathology in Alzheimer's disease. Exp Gerontol. 2010;45:75-9. 
18. Lucas JA, Ivnik RJ, Smith GE, Ferman TJ, Willis FB, Petersen RC, Graff-Radford NR. Mayo's Older African Americans Normative Studies: norms for Boston Naming Test, Controlled Oral Word Association, Category Fluency, Animal Naming, Token Test, WRAT-3 Reading, Trail Making Test, Stroop Test, and Judgment of Line Orientation. Clin Neuropsychol. 2005;19:243-69.

19. Machulda MM, Ivnik RJ, Smith GE, Ferman TJ, Boeve BF, Knopman D, Petersen RC, Tangalos EG. Mayo's Older Americans Normative Studies: Visual Form Discrimination and copy trial of the Rey-Osterrieth Complex Figure. J Clin Exp Neuropsychol. 2007;29:377-84.

20. Hebert LE, Scherr PA, Bennett DA, Bienias JL, Wilson RS, Morris MC, Evans DA. Blood pressure and late-life cognitive function change: a biracial longitudinal population study. Neurology. 2004;62:2021-4.

21. Crane PK, Narasimhalu K, Gibbons LE, Pedraza O, Mehta KM, Tang Y, Manly JJ, Reed BR, Mungas DM. Composite scores for executive function items: demographic heterogeneity and relationships with quantitative magnetic resonance imaging. J Int Neuropsychol Soc. 2008;14:746-59.

22. Crane PK, Carle A, Gibbons LE, Insel P, Mackin RS, Gross A, Jones RN, Mukherjee S, Curtis SM, Harvey D, et al. Development and assessment of a composite score for memory in the Alzheimer's Disease Neuroimaging Initiative (ADNI). Brain Imaging Behav. 2012;6:502-16.

23. Hu WT, Watts KD, Tailor P, Nguyen TP, Howell JC, Lee RC, Seyfried NT, Gearing M, Hales CM, Levey Al, et al. CSF complement 3 and factor $\mathrm{H}$ are staging biomarkers in Alzheimer's disease. Acta Neuropathol Commun. 2016;4:14.

24. Petersen RC, Smith GE, Waring SC, Ivnik RJ, Tangalos EG, Kokmen E. Mild cognitive impairment: clinical characterization and outcome. Arch Neurol. 1999;56:303-8.

25. Albert MS, DeKosky ST, Dickson D, Dubois B, Feldman HH, Fox NC, Gamst A Holtzman DM, Jagust WJ, Petersen RC, et al. The diagnosis of mild cognitive impairment due to Alzheimer's disease: recommendations from the National Institute on Aging and Alzheimer's Association Workgroup. Alzheimers Dement. 2011;7:270-9.

26. McKhann G, Drachman D, Folstein M, Katzman R, Price D, Stadlan EM. Clinical diagnosis of Alzheimer's disease: report of the NINCDSADRDA Work Group under the auspices of Department of Health and Human Services Task Force on Alzheimer's Disease. Neurology. 1984; 34:939-44.

27. Dubois $B$, Feldman HH, Jacova $C$, DeKosky ST, Barberger-Gateau $P$ Cummings J, Delacourte A, Galasko D, Gauthier S, Jicha G, et al. Research criteria for the diagnosis of Alzheimer's disease: revising the NINCDS-ADRDA criteria. Lancet Neurol. 2007;6:734-46.

28. Lucas JA, Ivnik RJ, Willis FB, Ferman TJ, Smith GE, Parfitt FC, Petersen RC, Graff-Radford NR. Mayo's Older African Americans Normative Studies: normative data for commonly used clinical neuropsychological measures. Clin Neuropsychol. 2005;19:162-83.

29. Shaw LM, Vanderstichele H, Knapik-Czajka M, Clark CM, Aisen PS, Petersen RC, Blennow K, Soares H, Simon A, Lewczuk P, et al. Cerebrospinal fluid biomarker signature in Alzheimer's disease neuroimaging initiative subjects. Ann Neurol. 2009:65:403-13.

30. Fischl B, Salat DH, Busa E, Albert M, Dieterich M, Haselgrove C, van der Kouwe A, Killiany R, Kennedy D, Klaveness S, et al. Whole brain segmentation: automated labeling of neuroanatomical structures in the human brain. Neuron. 2002;33:341-55

31. Carmichael O, Schwarz C, Drucker D, Fletcher E, Harvey D, Beckett L, Jack Jr CR, Weiner M, DeCarli C. Longitudinal changes in white matter disease and cognition in the first year of the Alzheimer disease neuroimaging initiative. Arch Neurol. 2010;67:1370-8.

32. Bateman RJ, Wen G, Morris JC, Holtzman DM. Fluctuations of CSF amyloid- $\beta$ levels: implications for a diagnostic and therapeutic biomarker. Neurology. 2007:68:666-9.

33. Hu WT, Watts KD, Shaw LM, Howell JC, Trojanowski JQ, Basra S, Glass JD, Lah JJ, Levey Al. CSF $\beta$-amyloid 1-42 - what are we measuring in Alzheimer's disease? Ann Clin Transl Neurol. 2015;2:131-9.

34. Jin SC, Carrasquillo MM, Benitez BA, Skorupa T, Carrell D, Patel D, Lincoln S, Krishnan S, Kachadoorian M, Reitz C, et al. TREM2 is associated with increased risk for Alzheimer's disease in African Americans. Mol Neurodegener. 2015;10:19.

35. Schneider AL, Sharrett AR, Gottesman RF, Coresh J, Coker L, Wruck L, Selnes OA, Deal J, Knopman D, Mosley TH. Normative data for 8 neuropsychological tests in older blacks and whites from the atherosclerosis risk in communities (ARIC) study. Alzheimer Dis Assoc Disord. 2015;29:32-44.
36. Fillenbaum GG, Heyman A, Huber MS, Ganguli M, Unverzagt FW. Performance of elderly African American and white community residents on the CERAD Neuropsychological Battery. J Int Neuropsychol Soc. 2001;7:502-9.

37. Jack Jr CR, Knopman DS, Jagust WJ, Shaw LM, Aisen PS, Weiner MW, Petersen RC, Trojanowski JQ. Hypothetical model of dynamic biomarkers of the Alzheimer's pathological cascade. Lancet Neurol. 2010;9:119-28.

38. Lee MJ, Seo SW, Na DL, Kim C, Park JH, Kim GH, Kim CH, Noh Y, Cho H, Kim $H J$, et al. Synergistic effects of ischemia and $\beta$-amyloid burden on cognitive decline in patients with subcortical vascular mild cognitive impairment. JAMA Psychiatry. 2014;71:412-22.

39. Steenland K, Goldstein FC, Levey A, Wharton W. A Meta-analysis of Alzheimer's disease incidence and prevalence comparing African-Americans and Caucasians. J Alzheimers Dis. 2016;50:71-6.

40. Hu WT, Watts K, Grossman M, Glass J, Lah JJ, Hales C, Shelnutt M, Van Deerlin V, Trojanowski JQ, Levey Al. Reduced CSF p-Tau 181 to Tau ratio is a biomarker for FTLD-TDP. Neurology. 2013;81:1945-52.

41. Evans W, Fung HC, Steele J, Eerola J, Tienari P, Pittman A, Silva R, Myers A, Vrieze FW, Singleton A, Hardy J. The tau H2 haplotype is almost exclusively Caucasian in origin. Neurosci Lett. 2004;369:183-5.

42. Kauwe JS, Cruchaga C, Mayo K, Fenoglio C, Bertelsen S, Nowotny P, Galimberti D, Scarpini E, Morris JC, Fagan AM, et al. Variation in MAPT is associated with cerebrospinal fluid tau levels in the presence of amyloid- $\beta$ deposition. Proc Natl Acad Sci U S A. 2008;105:8050-4.

43. Scheible M, Just R, Sturk-Andreaggi K, Saunier J, Parson W, Parsons T, Coble $\mathrm{M}$, Irwin J. The mitochondrial landscape of African Americans: an examination of more than 2500 control region haplotypes from 22 U.S. locations. Forensic Sci Int Genet. 2016;22:139-48.

\section{Submit your next manuscript to BioMed Central and we will help you at every step:}

- We accept pre-submission inquiries

- Our selector tool helps you to find the most relevant journal

- We provide round the clock customer support

- Convenient online submission

- Thorough peer review

- Inclusion in PubMed and all major indexing services

- Maximum visibility for your research

Submit your manuscript at www.biomedcentral.com/submit
C Biomed Central 